\title{
CONVERGENCE OF EQUILIBRIA OF PLANAR THIN ELASTIC BEAMS
}

\author{
M.G. MORA, S. MÜLLER, AND M.G. SCHULTZ
}

\begin{abstract}
We consider a thin elastic strip $\Omega_{h}=(0, L) \times(-h / 2, h / 2)$, and we show that stationary points of the nonlinear elastic energy (per unit height) $E^{h}(v)=\frac{1}{h} \int_{\Omega_{h}}\left(W(\nabla v)-h^{2} g\left(x_{1}\right) \cdot v\right) d x$ whose energy is bounded by $C h^{2}$ converge to stationary points of the Euler-Bernoulli functional $J_{2}(\bar{y})=\int_{0}^{L}\left(\frac{1}{24} E \kappa^{2}-\right.$ $g \cdot \bar{y}) d x_{1}$ where $\bar{y}:(0, L) \rightarrow \mathbb{R}^{2}$, with $\bar{y}^{\prime}=\left(\begin{array}{c}\cos \theta \\ \sin \theta\end{array}\right)$, and where $\kappa=\theta^{\prime}$. This corresponds to the equilibrium equation $-\frac{1}{12} E \theta^{\prime \prime}+\tilde{g} \cdot\left(\begin{array}{c}-\sin \theta \\ \cos \theta\end{array}\right)=0$, where $\tilde{g}$ is the primitive of $g$. The proof uses the rigidity estimate for low-energy deformations [4] and a compensated compactness argument in a singular geometry. In addition, possible concentration effects are ruled out by a careful truncation argument.
\end{abstract}

Keywords: dimension reduction, nonlinear elasticity, thin beams, equilibrium configurations

2000 Mathematics Subject Classification: $74 \mathrm{~K} 10$

\section{INTRODUCTION AND MAIN RESULT}

The relation between three-dimensional nonlinear elasticity and theories for lowerdimensional objects such as rods, beams, membranes, plates, and shells has been an outstanding question since the very beginning of research in elasticity. In fact, there is a large variety of lower-dimensional theories. They are usually obtained by making certain strong a-priori assumptions on the form of the solutions of the full three-dimensional problem, and hence their rigorous range of validity is typically unclear. As highlighted already in the work of Fritz John, the geometric nonlinearity in elasticity, i.e., the invariance of the elastic energy under rotations, is one of the key points. In particular, thin elastic objects can undergo large rotations even under small loads, and this prevents any analysis based on a naïve linearization. The first rigorous results were only obtained in the early 90's using a variational approach that guarantees convergence of minimizers to a suitable limit problem. In this paper, we discuss the convergence of possibly non-minimizing stationary points of the elastic energy functional.

To set the stage, let us first review the variational setting. Consider a cylindrical domain $\Omega_{h}=S \times\left(-\frac{h}{2}, \frac{h}{2}\right)$ where $S$ is a bounded subset of $\mathbb{R}^{2}$ with Lipschitz boundary. To a deformation $v: \Omega_{h} \rightarrow \mathbb{R}^{3}$, we associate the elastic energy (per unit height)

$$
E^{h}(v)=\frac{1}{h} \int_{\Omega_{h}} W(\nabla v) d z
$$


We assume that the stored-energy density $W$ satisfies the following conditions:

$$
\begin{aligned}
& W(R F)=W(F) \quad \forall R \in S O(3) \quad \text { (frame indifference), } \\
& W=0 \quad \text { on } S O(3), \\
& W(F) \geq c \operatorname{dist}^{2}(F, S O(3)), \quad c>0, \\
& W \text { is } C^{2} \text { in a neighbourhood of } S O(3) .
\end{aligned}
$$

Here, $S O(3)$ denotes the group of proper rotations. The frame indifference implies the existence of a function $\tilde{W}$ defined on symmetric matrices such that $W(\nabla v)=$ $\tilde{W}\left((\nabla v)^{T} \nabla v\right)$, i.e., the elastic energy depends only on the pull-back metric of $v$.

For the discussion of the limiting behavior of $E^{h}$ as $h \rightarrow 0$, it is convenient to rescale $\Omega_{h}$ to a fixed domain $\Omega=S \times\left(-\frac{1}{2}, \frac{1}{2}\right)$ by a change of variables, $z=$ $\left(x_{1}, x_{2}, h x_{3}\right)$ and $y(x)=v(z)$. With the notation

$$
\nabla_{h} y=\left(\partial_{1} y, \partial_{2} y, \frac{1}{h} \partial_{3} y\right)=\left(\nabla^{\prime} y, \frac{1}{h} \partial_{3} y\right),
$$

we thus have

$$
E^{h}(v)=I^{h}(y)=\int_{\Omega} W\left(\nabla_{h} y\right) d x .
$$

The variational approach leads to a hierarchy of limiting theories depending on the scaling of $I^{h}$. More precisely, as $h \rightarrow 0$

$$
\frac{1}{h^{\beta}} I^{h} \stackrel{\Gamma}{\longrightarrow} I_{\beta}
$$

in the sense of $\Gamma$-convergence. This implies, roughly speaking, that minimizers of $I^{h}$ (subject to suitable boundary conditions or body forces) converge to minimizers of $I_{\beta}$, provided $I^{h}$ evaluated on the minimizers is bounded by $C h^{\beta}$. $\Gamma$-convergence was first established by LeDret and Raoult for $\beta=0$ (see [6]), then for all $\beta \geq 2$ in [4, 5]. (see also [11, 12, for results for $\beta=2$ under additional conditions). For $0<\beta<5 / 3$ convergence was recently obtained by Conti and Maggi in [2], see also [1. The exponent $\beta=5 / 3$ is conjectured to be relevant for the crumpling of elastic sheets (see [8, 14, 2]).

Here, we focus on the case $\beta=2$, which leads to Kirchhoff's geometrically nonlinear bending theory. The natural class $\mathcal{A}$ of admissible functions for the limit problem is given by isometric $W^{2,2}$ immersions from $S$ into $\mathbb{R}^{3}$, i.e.,

$$
\mathcal{A}:=\left\{y \in W^{2,2}\left(\Omega, \mathbb{R}^{3}\right): \partial_{3} y=0,\left(\nabla^{\prime} y\right)^{T} \nabla^{\prime} y=I d\right\} .
$$

The limiting energy functional is

$$
I_{2}(y)= \begin{cases}\frac{1}{24} \int_{S} Q_{2}(A) d x_{1} d x_{2} & \text { if } y \in \mathcal{A}, \\ +\infty & \text { else. }\end{cases}
$$

Here, $A$ is the second fundamental form, and $Q_{2}$ is a quadratic form that can be computed from the linearization $D^{2} W(I d)$ of the $3 \mathrm{~d}$ energy at the identity. If $W(F)=\frac{1}{2} \operatorname{dist}^{2}(F, S O(3))$, then simply $Q_{2}(A)=|A|^{2}$.

In this paper, we consider the convergence of equilibria for the case $\beta=2$. Instead of treating the full problem of a reduction from $3 \mathrm{~d}$ to $2 \mathrm{~d}$, we focus on the simpler case $2 \mathrm{~d}$ to $1 \mathrm{~d}$. Thus, we start from a thin strip

$$
\Omega_{h}=(0, L) \times\left(-\frac{h}{2}, \frac{h}{2}\right),
$$


and after the rescaling $\left(z_{1}, z_{2}\right)=\left(x_{1}, h x_{2}\right), \nabla_{h}=\left(\partial_{1}, \frac{1}{h} \partial_{2}\right)$, we consider the functional

$$
J^{h}(y)=\int_{\Omega}\left(W\left(\nabla_{h} y\right)-h^{2} g\left(x_{1}\right) \cdot y\right) d x
$$

The corresponding $\Gamma$-limit is given by

$$
J_{2}(\bar{y})=\int_{0}^{L}\left(\frac{1}{24} E \kappa^{2}-g \cdot \bar{y}\right) d x_{1},
$$

where

$$
\bar{y}:(0, L) \rightarrow \mathbb{R}^{2}, \quad \bar{y}^{\prime}=\left(\begin{array}{c}
\cos \theta \\
\sin \theta
\end{array}\right), \quad \kappa=\theta^{\prime} .
$$

The functional $J_{2}$ takes the value $+\infty$, if $\bar{y}$ is not of the above form (here we took the liberty to identify maps on $\Omega$ which are independent of $x_{2}$ with maps on $\left.(0, L)\right)$. It is convenient to fix one endpoint by requiring $\bar{y}(0)=0$. Integrating the linear term by parts, we obtain the following Euler-Lagrange equation corresponding to the limit functional

$$
-\frac{1}{12} E \theta^{\prime \prime}+\tilde{g} \cdot\left(\begin{array}{c}
-\sin \theta \\
\cos \theta
\end{array}\right)=0, \quad \tilde{g}\left(x_{1}\right):=\int_{L}^{x_{1}} g(\xi) d \xi
$$

The main result of the paper is the following.

Theorem 1.1. Assume (1.1)-(1.4), the energy $W$ is differentiable, and the derivative $D W$ is globally Lipschitz. Let $\left(y^{(h)}\right)$ be a sequence of stationary points of $J^{h}$, subject to the boundary condition $y^{(h)}\left(0, x_{2}\right)=\left(0, h x_{2}\right)$ at $x_{1}=0$ and to natural boundary conditions on the remaining boundaries. Assume further

$$
\int_{\Omega} W\left(\nabla_{h} y^{(h)}\right) \leq C h^{2}
$$

Then, up to subsequences,

$$
y^{(h)} \rightarrow \bar{y} \quad \text { in } W^{1,2}\left(\Omega ; \mathbb{R}^{2}\right),
$$

as $h \rightarrow 0$. The limit function $\bar{y}$ satisfies

$$
\partial_{1} \bar{y}=\left(\begin{array}{c}
\cos \theta \\
\sin \theta
\end{array}\right), \quad \partial_{2} \bar{y}=0
$$

and $\theta$ satisfies (1.6) and

$$
\theta(0)=0, \quad \theta^{\prime}(L)=0 .
$$

Remark 1.2. An easy application of Poincaré's inequality shows that the estimate (1.7) automatically holds for minimizers.

Remark 1.3. In [9, Mielke uses a center manifold approach to compare solutions in a thin strip to a $1 \mathrm{~d}$ problem. His approach already works for finite $h$, but requires that the nonlinear strain $\left(\nabla_{h} y\right)^{T} \nabla_{h} y$ is $L^{\infty}$-close to the identity. Applied forces $g$ are also difficult to handle.

One key idea in the proof of Theorem 1.1 is to replace the use of comparison functions in $\Gamma$-convergence by a compensated compactness argument (see Steps 6 and 7 in the next section). To set the stage for this argument, we use the quantitative rigidity estimate of 4 to introduce suitable strain-like and stress-like variables $G^{(h)}$ and $E^{(h)}$, which are almost curl-free and divergence-free, respectively (see Steps 2 and 3 below). To control possible concentration effects, we use a truncation argument, see Section 3. 


\section{PROOF}

Let $\left(y^{(h)}\right)$ be a sequence of stationary points of $J^{h}$, i.e., suppose that

$$
\int_{\Omega}\left(D W\left(\nabla_{h} y^{(h)}\right): \nabla_{h} \psi-h^{2} g \cdot \psi\right) d x=0
$$

for every $\psi \in W^{1,2}\left(\Omega ; \mathbb{R}^{2}\right)$ with $\psi=0$ on $\left\{x_{1}=0\right\}$. Assume further estimate (1.7). Step 1. Decomposition of the deformation gradients in rotation and strain.

By Proposition 4.1, we can construct a sequence $\left(R^{(h)}\right) \subset C^{\infty}\left((0, L) ; \mathbb{M}^{2 \times 2}\right)$ such that for every $x_{1} \in(0, L), R^{(h)}\left(x_{1}\right) \in S O(2)$ and

$$
\begin{gathered}
\left\|\nabla_{h} y^{(h)}-R^{(h)}\right\|_{L^{2}} \leq C h, \\
\left\|\left(R^{(h)}\right)^{\prime}\right\|_{L^{2}}+h\left\|\left(R^{(h)}\right)^{\prime \prime}\right\|_{L^{2}} \leq C, \\
\left|R^{(h)}(0)-I d\right| \leq C \sqrt{h} .
\end{gathered}
$$

By (2.3), there exists $R \in W^{1,2}\left((0, L) ; \mathbb{M}^{2 \times 2}\right)$ such that up to subsequences $R^{(h)}$ converges to $R$ weakly in $W^{1,2}\left((0, L) ; \mathbb{M}^{2 \times 2}\right)$, hence uniformly in $L^{\infty}\left((0, L) ; \mathbb{M}^{2 \times 2}\right)$. Thus $R\left(x_{1}\right) \in S O(2)$ for every $x_{1} \in(0, L)$. Moreover, estimate (2.2) implies that

$$
\nabla_{h} y^{(h)} \rightarrow R \text { strongly in } L^{2}\left(\Omega ; \mathbb{M}^{2 \times 2}\right) .
$$

In particular, $\partial_{2} y^{(h)} \rightarrow 0$ and thus

$$
\nabla y^{(h)} \rightarrow R e_{1} \otimes e_{1} \quad \text { strongly in } L^{2}\left(\Omega ; \mathbb{M}^{2 \times 2}\right) .
$$

Since $\left|y^{(h)}\left(0, x_{2}\right)\right| \leq h \rightarrow 0$, we deduce from Poincaré's inequality that $y^{(h)} \rightarrow \bar{y}$ strongly in $W^{1,2}\left(\Omega ; \mathbb{R}^{2}\right)$ and that $\bar{y}$ satisfies $\partial_{1} \bar{y}=R e_{1}, \partial_{2} \bar{y}=0$ a.e. in $\Omega$. Thus, (1.8) and (1.9) are proved.

We now make use of the approximated sequence of rotations $R^{(h)}$ in order to decompose the deformation gradients as

$$
\nabla_{h} y^{(h)}=R^{(h)}\left(I d+h G^{(h)}\right) .
$$

The $G^{(h)}: \Omega \rightarrow \mathbb{M}^{2 \times 2}$ are bounded in $L^{2}\left(\Omega ; \mathbb{M}^{2 \times 2}\right)$ by (2.2). Thus, up to extracting a subsequence, we can assume

$$
G^{(h)} \rightarrow G \quad \text { weakly in } L^{2}\left(\Omega ; \mathbb{M}^{2 \times 2}\right)
$$

for some $G \in L^{2}\left(\Omega ; \mathbb{M}^{2 \times 2}\right)$.

\section{Step 2. Consequences of the compatibility of the strain.}

Up to the factor $\left(R^{(h)}\right)^{T}$, the strains $G^{(h)}$ are essentially scaled gradients. This has some important consequences on the form of the limit strain $G$. To deduce these properties, it is convenient to introduce a sequence of auxiliary functions $z^{(h)}: \Omega \rightarrow$ $\mathbb{R}^{2}$ defined as

$$
z^{(h)}(x):=\frac{1}{h} y^{(h)}(x)-\frac{1}{h} \int_{0}^{x_{1}} R^{(h)}(s) e_{1} d s-x_{2} R^{(h)}\left(x_{1}\right) e_{2} .
$$

By the definition (2.6) of $G^{(h)}$,

$$
\begin{aligned}
\nabla_{h} z^{(h)} & =\frac{1}{h}\left(\nabla_{h} y^{(h)}-R^{(h)}\right)-x_{2}\left(R^{(h)}\right)^{\prime} e_{2} \otimes e_{1} \\
& =R^{(h)}\left(G^{(h)}-x_{2}\left(R^{(h)}\right)^{T}\left(R^{(h)}\right)^{\prime} e_{2} \otimes e_{1}\right) .
\end{aligned}
$$


Since $R^{(h)} \in S O(2)$, there exist $\theta^{(h)} \in C^{\infty}(0, L)$ such that

$$
R^{(h)}=\left(\begin{array}{cc}
\cos \theta^{(h)} & -\sin \theta^{(h)} \\
\sin \theta^{(h)} & \cos \theta^{(h)}
\end{array}\right) .
$$

Then $\left(R^{(h)}\right)^{T}\left(R^{(h)}\right)^{\prime} e_{2}=-\left(\theta^{(h)}\right)^{\prime} e_{1}$, and equality (2.9) can be rewritten as

$$
\nabla_{h} z^{(h)}=R^{(h)}\left(G^{(h)}+x_{2}\left(\theta^{(h)}\right)^{\prime} e_{1} \otimes e_{1}\right) .
$$

Now, recall that $R^{(h)}$ converges uniformly to $R, G^{(h)}$ converges weakly to $G$ in $L^{2}\left(\Omega ; \mathbb{M}^{3 \times 3}\right)$, and $\left(\theta^{(h)}\right)^{\prime}$ converges weakly to $\theta^{\prime}$ in $L^{2}(0, L)$, where $\theta$ satisfies (1.9). From these properties it follows that

$$
\nabla_{h} z^{(h)} \rightarrow R\left(G+x_{2} \theta^{\prime} e_{1} \otimes e_{1}\right) \quad \text { weakly in } L^{2}\left(\Omega ; \mathbb{M}^{2 \times 2}\right) .
$$

The definition of $z^{(h)}$ and (2.4) yield $\left|z^{(h)}\left(0, x_{2}\right)\right| \leq C \sqrt{h}$. Hence Poincaré's inequality shows that $z^{(h)}$ converges weakly in $W^{1,2}\left(\Omega ; \mathbb{R}^{2}\right)$. The limit function $z$ satisfies

$$
R^{T} \partial_{1} z=G e_{1}+x_{2} \theta^{\prime} e_{1}, \quad \partial_{2} z=0 \quad \text { a.e. in } \Omega .
$$

In particular, $z$ does not depend on $x_{2}$, and thus, by the first equality in (2.12), the vector $G e_{1}$ is linear in $x_{2}$.

Let $\hat{G}:(0, L) \rightarrow \mathbb{M}^{2 \times 2}$ be the first moment of $G$ defined by

$$
\hat{G}\left(x_{1}\right):=\int_{-\frac{1}{2}}^{\frac{1}{2}} x_{2} G(x) d x_{2} .
$$

As $R$ and $z$ are independent of $x_{2}$, we deduce from (2.12) that

$$
\hat{G}_{11}=-\frac{1}{12} \theta^{\prime}, \quad \hat{G}_{21}=0 \quad \text { a.e. in }(0, L) .
$$

Step 3. Consequences of the Euler-Lagrange equations.

Let $E^{(h)}: \Omega \rightarrow \mathbb{M}^{2 \times 2}$ be the scaled stress defined by

$$
E^{(h)}:=\frac{1}{h} D W\left(I d+h G^{(h)}\right) .
$$

Since $D W$ is Lipschitz continuous and the $G^{(h)}$ are bounded in $L^{2}\left(\Omega ; \mathbb{M}^{2 \times 2}\right)$, the functions $E^{(h)}$ are bounded in $L^{2}\left(\Omega ; \mathbb{M}^{2 \times 2}\right)$. By Proposition 4.2 , we have

$$
E^{(h)} \rightarrow E:=\mathcal{L} G \quad \text { weakly in } L^{2}\left(\Omega ; \mathbb{M}^{2 \times 2}\right),
$$

where the linear map $\mathcal{L}$ on the matrix space is given by $\mathcal{L}:=D^{2} W(I d)$. We note in particular that $E$ is symmetric since, by frame indifference, $\mathcal{L} F=\mathcal{L} \operatorname{sym} F$ and $\mathcal{L} F=(\mathcal{L} F)^{T}$ for all $F \in \mathbb{M}^{2 \times 2}$.

By the decomposition (2.6) and frame indifference of $W$, we obtain

$$
D W\left(\nabla_{h} y^{(h)}\right)=R^{(h)} D W\left(I d+h G^{(h)}\right)=h R^{(h)} E^{(h)} .
$$

The Euler-Lagrange equations (2.1) can be written in terms of the stresses $E^{(h)}$ :

$$
\int_{\Omega}\left(R^{(h)} E^{(h)}: \nabla_{h} \psi-h g \cdot \psi\right) d x=0
$$


for every $\psi \in W^{1,2}\left(\Omega ; \mathbb{R}^{2}\right)$ with $\psi=0$ on $\left\{x_{1}=0\right\}$. Multiplying (2.16) by $h$ and passing to the limit as $h \rightarrow 0$, we find

$$
\int_{\Omega} R E e_{2} \cdot \partial_{2} \psi d x=0
$$

for every $\psi \in W^{1,2}\left(\Omega ; \mathbb{R}^{2}\right)$ with $\psi=0$ on $\left\{x_{1}=0\right\}$. This yields $R E e_{2}=0$ a.e. in $\Omega$ and hence $E e_{2}=0$ a.e. in $\Omega$. Therefore, as $E$ is symmetric, we conclude that

$$
E(x)=E_{11}(x) e_{1} \otimes e_{1}
$$

for a.e. $x \in \Omega$.

Step 4. Symmetry properties of $E^{(h)}$.

Since $W$ is frame indifferent, the matrix $D W(F) F^{T}$ is symmetric. Choosing $F=$ $I d+h G^{(h)}$, we deduce that

$$
E^{(h)}-\left(E^{(h)}\right)^{T}=-h\left(E^{(h)}\left(G^{(h)}\right)^{T}-G^{(h)}\left(E^{(h)}\right)^{T}\right) .
$$

Using the boundedness of $E^{(h)}$ and $G^{(h)}$ in $L^{2}\left(\Omega ; \mathbb{M}^{2 \times 2}\right)$, we have in particular the estimate

$$
\left\|E_{12}^{(h)}-E_{21}^{(h)}\right\|_{L^{1}} \leq C h
$$

Step 5. Moments of the Euler-Lagrange equations.

Let us introduce the zeroth and the first moment of the stress $E^{(h)}$,

$$
\bar{E}^{(h)}\left(x_{1}\right):=\int_{-\frac{1}{2}}^{\frac{1}{2}} E^{(h)}(x) d x_{2}, \quad \hat{E}^{(h)}\left(x_{1}\right):=\int_{-\frac{1}{2}}^{\frac{1}{2}} x_{2} E^{(h)}(x) d x_{2},
$$

for every $x_{1} \in(0, L)$. In the following, we will derive the equations satisfied by these moments.

Let $\varphi \in C^{\infty}\left([0, L] ; \mathbb{R}^{2}\right)$ be such that $\varphi(0)=0$. Using $\varphi$ as a test function for the Euler-Lagrange equation (2.16), we obtain

$$
\int_{\Omega}\left(R^{(h)} E^{(h)} e_{1} \cdot \varphi^{\prime}-h g \cdot \varphi\right) d x=0 .
$$

As $R^{(h)}, \varphi$, and $g$ depend only on the variable $x_{1}$, this equality is equivalent to

$$
\int_{0}^{L}\left(R^{(h)} \bar{E}^{(h)} e_{1} \cdot \varphi^{\prime}-h g \cdot \varphi\right) d x_{1}=0 .
$$

This equation holds for every $\varphi \in C^{\infty}\left([0, L] ; \mathbb{R}^{2}\right)$ with $\varphi(0)=0$, and hence we deduce that

$$
\bar{E}^{(h)} e_{1}=-h\left(R^{(h)}\right)^{T} \tilde{g} \quad \text { a.e. in }(0, L),
$$

where $\tilde{g}$ is the primitive of $g$ defined in (1.6). By passing to the limit, we obtain

$$
\bar{E} e_{1}=0 \quad \text { a.e. in }(0, L) \text {. }
$$

As for the first moment, let $\varphi \in C^{\infty}\left([0, L] ; \mathbb{R}^{2}\right)$ be again such that $\varphi(0)=0$. Using $\psi(x):=x_{2} \varphi\left(x_{1}\right)$ as a test function in (2.16), we now obtain

$$
\int_{\Omega}\left(x_{2} R^{(h)} E^{(h)} e_{1} \cdot \varphi^{\prime}+\frac{1}{h} R^{(h)} E^{(h)} e_{2} \cdot \varphi-h x_{2} g \cdot \varphi\right) d x=0 .
$$

Upon integration with respect to $x_{2}$, this equation becomes

$$
\int_{0}^{L}\left(R^{(h)} \hat{E}^{(h)} e_{1} \cdot \varphi^{\prime}+\frac{1}{h} R^{(h)} \bar{E}^{(h)} e_{2} \cdot \varphi\right) d x_{1}=0 .
$$


We can choose $\varphi$ to be of the form $\varphi=\phi R^{(h)} e_{1}$ with $\phi \in C^{\infty}([0, L])$ and $\phi(0)=0$. Hence

$$
\int_{0}^{L}\left(\hat{E}_{11}^{(h)} \phi^{\prime}+\hat{E}^{(h)} e_{1} \cdot \phi\left(R^{(h)}\right)^{T}\left(R^{(h)}\right)^{\prime} e_{1}+\frac{1}{h} \bar{E}_{12}^{(h)} \phi\right) d x_{1}=0 .
$$

Wtih the identity $\left(R^{(h)}\right)^{T}\left(R^{(h)}\right)^{\prime} e_{1}=\left(\theta^{(h)}\right)^{\prime} e_{2}$, this expression reduces to

$$
\int_{0}^{L}\left(\hat{E}_{11}^{(h)} \phi^{\prime}+\left(\theta^{(h)}\right)^{\prime} \hat{E}_{21}^{(h)} \phi+\frac{1}{h} \bar{E}_{12}^{(h)} \phi\right) d x_{1}=0 .
$$

From the estimate (2.20) and the identity (2.21), we infer an $L^{1}(0, L)$-bound on $\frac{1}{h} \bar{E}_{12}^{(h)}$. Since $\left(\theta^{(h)}\right)^{\prime}$ and $\hat{E}_{21}^{(h)}$ are bounded in $L^{2}(0, L),\left(\theta^{(h)}\right)^{\prime} \hat{E}_{21}^{(h)}$ is clearly bounded in $L^{1}(0, L)$. Therefore, equation (2.23) implies that

$$
\left\|\partial_{1} \hat{E}_{11}^{(h)}\right\|_{L^{1}} \leq C, \quad \hat{E}_{11}^{(h)}(L)=0 .
$$

Hence, the sequence $\hat{E}_{11}^{(h)}$ is strongly compact in $L^{p}(0, L)$ for every $p<\infty$.

Step 6. Convergence of the energy by the div-curl lemma.

The strong compactness of the sequence $\left(\hat{E}_{11}^{(h)}\right)$ allows us to pass to the limit in the energy integral

$$
\frac{1}{h^{2}} \int_{\Omega} D W\left(I d+h G^{(h)}\right): h G^{(h)} d x=\int_{\Omega} E^{(h)}: G^{(h)} d x .
$$

The limit is obtained by exploiting the div-curl structure of the product $E^{(h)}: G^{(h)}$. By the Euler-Lagrange equation (2.16), the scaled divergence of $R^{(h)} E^{(h)}$ is infinitesimal in $L^{2}\left(\Omega ; \mathbb{R}^{2}\right)$ as $h \rightarrow 0$, while by the decomposition (2.10), the matrix $R^{(h)} G^{(h)}$ has essentially the structure of a scaled gradient.

Let $\varphi \in C^{\infty}([0, L])$ with $\varphi(0)=0$. Using formula (2.10), we have

$$
\begin{aligned}
& \int_{\Omega} \varphi E^{(h)}: G^{(h)} d x=\int_{\Omega} \varphi R^{(h)} E^{(h)}: R^{(h)} G^{(h)} d x \\
& =\int_{\Omega} \varphi R^{(h)} E^{(h)}: \nabla_{h} z^{(h)} d x-\int_{\Omega} \varphi E_{11}^{(h)} x_{2}\left(\theta^{(h)}\right)^{\prime} d x .
\end{aligned}
$$

We deduce from the Euler-Lagrange equation (2.16), applied with $\psi=\varphi z^{(h)}$, that

$$
\int_{\Omega} \varphi R^{(h)} E^{(h)}: \nabla_{h} z^{(h)} d x=h \int_{\Omega} \varphi g \cdot z^{(h)} d x-\int_{\Omega} \varphi^{\prime} R^{(h)} E^{(h)} e_{1} \cdot z^{(h)} d x .
$$

Since $z^{(h)}$ converges strongly to $z$ in $L^{2}\left(\Omega ; \mathbb{R}^{2}\right)$, we can pass to the limit in the above formula and obtain

$$
\begin{aligned}
\lim _{h \rightarrow 0} \int_{\Omega} \varphi R^{(h)} E^{(h)}: \nabla_{h} z^{(h)} d x & =-\int_{\Omega} \varphi^{\prime} R E e_{1} \cdot z d x \\
& =-\int_{0}^{L} \varphi^{\prime} R \bar{E} e_{1} \cdot z d x_{1}=0,
\end{aligned}
$$

where the last two equalities follow from the independence of $z$ of $x_{2}$ and the identity (2.22).

As for the last term in (2.25), we have

$$
\int_{\Omega} \varphi E_{11}^{(h)} x_{2}\left(\theta^{(h)}\right)^{\prime} d x=\int_{0}^{L} \varphi \hat{E}_{11}^{(h)}\left(\theta^{(h)}\right)^{\prime} d x_{1}
$$


and, using the strong convergence of $\hat{E}_{11}^{(h)}$ proved at the end of Step 5, we deduce that

$$
\lim _{h \rightarrow 0} \int_{\Omega} \varphi E_{11}^{(h)} x_{2}\left(\theta^{(h)}\right)^{\prime} d x=\int_{0}^{L} \varphi \hat{E}_{11} \theta^{\prime} d x_{1} .
$$

From the first equality in (2.12), we infer that

$$
\int_{0}^{L} \varphi \hat{E}_{11} \theta^{\prime} d x_{1}=\int_{\Omega} \varphi E_{11} x_{2} \theta^{\prime} d x=\int_{\Omega} \varphi E_{11} R^{T} z^{\prime} \cdot e_{1} d x-\int_{\Omega} \varphi E_{11} G_{11} d x .
$$

Using (2.22) and (2.18), the previous equality yields

$$
\begin{aligned}
\int_{0}^{L} \varphi \hat{E}_{11} \theta^{\prime} d x_{1} & =\int_{0}^{L} \varphi \bar{E}_{11} R^{T} z^{\prime} \cdot e_{1} d x_{1}-\int_{\Omega} \varphi E_{11} G_{11} d x \\
& =-\int_{\Omega} \varphi E: G d x
\end{aligned}
$$

Finally combining equations (2.25) -(2.28), we obtain convergence of the energies

$$
\lim _{h \rightarrow 0} \int_{\Omega} \varphi E^{(h)}: G^{(h)} d x=\int_{\Omega} \varphi E: G d x
$$

for every $\varphi \in C^{\infty}([0, L])$ with $\varphi(0)=0$.

Step 7. Strong convergence of the symmetric part of $G^{(h)}$.

In order to emphasize the structure of the argument, we first conclude the proof under the additional assumption

$$
\lim _{h \rightarrow 0} h\left\|G^{(h)}\right\|_{L^{\infty}}=0 .
$$

This assumption will allow us to replace $E^{(h)}$ in (2.29) by $\mathcal{L} G^{(h)}$. Since we already know that $E=\mathcal{L} G$ and since $\mathcal{L}$ is positive definite on symmetric matrices, we can conclude strong convergence of $G^{(h)}$ and hence of $E^{(h)}$ (away from $x_{1}=0$ ). Using this strong convergence, we can easily pass to the limit in (2.23), using formula (2.19) for the skew-symmetric part of $E^{(h)}$ and the good control on $E_{21}^{(h)}$. We will show in the next section how assumption (2.30) can be avoided through the use of a careful truncation argument.

A Taylor expansion of $D W$ around the identity matrix yields

$$
E^{(h)}=\frac{1}{h} D W\left(I d+h G^{(h)}\right)=\mathcal{L} G^{(h)}+\frac{1}{h} \eta\left(h G^{(h)}\right),
$$

where $|\eta(A)| /|A| \rightarrow 0$, as $|A| \rightarrow 0$. For every $t>0$, we define

$$
\omega(t):=\sup \left\{\frac{|\eta(A)|}{|A|}:|A| \leq t\right\} .
$$

Then $\omega(t) \rightarrow 0$, as $t \rightarrow 0^{+}$. Using the expansion (2.31), we obtain

$$
\left|E^{(h)}: G^{(h)}-\mathcal{L} G^{(h)}: G^{(h)}\right| \leq \frac{1}{h}\left|\eta\left(h G^{(h)}\right)\right|\left|G^{(h)}\right| \leq \omega\left(h\left\|G^{(h)}\right\|_{L^{\infty}}\right)\left|G^{(h)}\right|^{2} .
$$

Assumption (2.30) implies that the last term in this inequality tends to zero in $L^{1}(\Omega)$, as $h \rightarrow 0$. By (2.29) and (2.15), we thus obtain for every $\varphi \in C^{\infty}([0, L])$ with $\varphi(0)=0$

$$
\lim _{h \rightarrow 0} \int_{\Omega} \varphi \mathcal{L} G^{(h)}: G^{(h)} d x=\int_{\Omega} \varphi \mathcal{L} G: G d x .
$$


From the assumptions on $W$, we obtain a constant $C>0$ such that

$$
\mathcal{L} A: A \geq C|\operatorname{sym} A|^{2}
$$

for every $A \in \mathbb{M}^{2 \times 2}$. This inequality, together with (2.7) and (2.32), implies that

$$
\lim _{h \rightarrow 0} C \int_{\Omega} \varphi\left|\operatorname{sym}\left(G^{(h)}-G\right)\right|^{2} d x \leq \lim _{h \rightarrow 0} \int_{\Omega} \varphi \mathcal{L}\left(G^{(h)}-G\right):\left(G^{(h)}-G\right) d x=0
$$

for every nonnegative $\varphi \in C^{\infty}([0, L])$ with $\varphi(0)=0$. In particular, we have

$$
\operatorname{sym} G^{(h)} \rightarrow \operatorname{sym} G \quad \text { strongly in } L^{2}\left((a, L) \times\left(-\frac{1}{2}, \frac{1}{2}\right) ; \mathbb{M}^{2 \times 2}\right)
$$

for every $a>0$. Since $\mathcal{L} A=\mathcal{L} \operatorname{sym} A$ for every $A \in \mathbb{M}^{2 \times 2}$, the Taylor expansion (2.31), together with (2.30) and (2.33), yields

$$
E^{(h)} \rightarrow E=\mathcal{L} \operatorname{sym} G \quad \text { strongly in } L^{2}\left((a, L) \times\left(-\frac{1}{2}, \frac{1}{2}\right) ; \mathbb{M}^{2 \times 2}\right)
$$

for every $a>0$.

Step 8. Derivation of the limit equation.

Due to the convergence (2.34), we can pass to the limit in (2.19) and obtain

$$
\frac{1}{h}\left(E^{(h)}-\left(E^{(h)}\right)^{T}\right) \rightarrow G E^{T}-E G^{T}
$$

weakly in $L^{1}\left((a, L) \times\left(-\frac{1}{2}, \frac{1}{2}\right) ; \mathbb{M}^{2 \times 2}\right)$ for every $a>0$. Note that by (2.18) one has

$$
G E^{T}-E G^{T}=E_{11}\left(G e_{1} \otimes e_{1}-e_{1} \otimes G e_{1}\right)=E_{11} G_{21}\left(e_{2} \otimes e_{1}-e_{1} \otimes e_{2}\right)
$$

and recall that $G_{21}$ is independent of $x_{2}$ by (2.12). Thus, by (2.35) and (2.22) we deduce that

$$
\frac{1}{h}\left(\bar{E}_{21}^{(h)}-\bar{E}_{12}^{(h)}\right) \rightarrow G_{21} \bar{E}_{11}=0 \quad \text { weakly in } L^{1}(a, L)
$$

for every $a>0$.

We now have all the necessary ingredients to derive the limit equation. It follows from (2.21) that $\frac{1}{h} \bar{E}_{21}^{(h)}$ converges to $-R^{T} \tilde{g} \cdot e_{2}$ strongly in $L^{2}(0, L)$. Combining this with (2.36), we deduce that $\frac{1}{h} \bar{E}_{12}^{(h)}$ converges to $-R^{T} \tilde{g} \cdot e_{2}$ weakly in $L^{1}(a, L)$ for every $a>0$. Using the strong convergence (2.34) and the fact that $\hat{E}_{21}=0$ by (2.18), we can pass to the limit in the equation (2.23) and conclude that

$$
\int_{0}^{L}\left(\hat{E}_{11} \varphi^{\prime}-R^{T} \tilde{g} \cdot e_{2} \varphi\right) d x_{1}=0
$$

for every $\varphi \in C^{\infty}([0, L])$ which vanishes on an interval $(0, a)$, with $a>0$. By approximation we see that the limiting equation holds for all $\varphi \in C^{\infty}([0, L])$ such that $\varphi(0)=0$.

Since $E=\mathcal{L} \operatorname{sym} G$ and $E=E_{11} e_{1} \otimes e_{1}$, we have

$$
\operatorname{sym} G=E_{11} \mathcal{L}^{-1}\left(e_{1} \otimes e_{1}\right),
$$

which yields

$$
G_{11}=E_{11} \mathcal{L}^{-1}\left(e_{1} \otimes e_{1}\right):\left(e_{1} \otimes e_{1}\right) .
$$

Using the representation formula (2.13), we deduce that

$$
-\frac{1}{12} \theta^{\prime}=\hat{E}_{11} \mathcal{L}^{-1}\left(e_{1} \otimes e_{1}\right):\left(e_{1} \otimes e_{1}\right) .
$$


Thus, combining this equality and (2.37), we conclude that (1.6) is satisfied with $E^{-1}=\mathcal{L}^{-1}\left(e_{1} \otimes e_{1}\right):\left(e_{1} \otimes e_{1}\right)$. Moreover, the natural boundary condition follows directly from (2.37), while (2.4) and the uniform convergence of $R^{(h)}$ imply $\theta(0)=0$.

\section{TRUnCATion AND COMPACTNESS}

Apart from some minor issues which are discussed in the next section, the main point is to remove the strong hypothesis $h\left\|G^{(h)}\right\|_{\infty} \rightarrow 0$ in the argument of Section 2. To achieve this, we will use a truncation argument. We first observe that the standard truncation result can also be applied to functions defined in thin rectangles, equivalently it can be applied to the scaled gradient $\nabla_{h}=\left(\partial_{1}, \frac{1}{h} \partial_{2}\right)$. Moreover by a good choice of the truncation parameter, the bad set where the truncation does not agree with the original function can be chosen to be particularly small (see Lemma 4.3 below).

Lemma 3.1. There exists a constant $C$ with the following property. For every $h>0$, every $A>a>0$ and every $u \in W^{1,2}\left(\Omega_{h} ; \mathbb{R}^{2}\right)$ there exist $\lambda \in[a, A]$ and $a$ function $v \in W^{1, \infty}\left(\Omega_{h} ; \mathbb{R}^{2}\right)$ such that

$$
\begin{gathered}
\|\nabla v\|_{L^{\infty}} \leq \lambda, \\
\lambda^{2} \mathcal{L}^{2}\left(\left\{x \in \Omega_{h}: u(x) \neq v(x)\right\}\right) \leq \frac{C}{\ln (A / a)} \int_{\Omega_{h}}|\nabla u|^{2} d x .
\end{gathered}
$$

For the proof, we refer to Section 4 . We also recall that

$$
\nabla u=\nabla v \quad \text { a.e. in the set }\left\{x \in \Omega_{h}: u(x)=v(x)\right\} .
$$

Conclusion of the proof of Theorem 1.1. Using a truncation, we first define functions $\tilde{y}^{(h)}$ such that the corresponding rescaled strains $\tilde{G}^{(h)}$ satisfy

$$
\lim _{h \rightarrow 0} h\left\|\tilde{G}^{(h)}\right\|_{L^{\infty}}=0 .
$$

We can then use a Taylor expansion as in Step 7 of the previous section to conclude that $\int \varphi \mathcal{L} \tilde{G}^{(h)}: \tilde{G}^{(h)} \approx \int \varphi \tilde{E}^{(h)}: \tilde{G}^{(h)}$. The crucial step is to show strong convergence of sym $\tilde{G}^{(h)}$ (away from $x_{1}=0$ ). It will be easy to see that $\tilde{G}^{(h)}$ and $G^{(h)}$ have the same weak limit. The main point is to get strong $L^{2}$ convergence of the truncated sequence $\tilde{G}^{(h)}$. We can adapt the compactness argument in Step 7 of the previous section to get convergence of the truncated sequence if we can show that

$$
\int_{\Omega} \varphi E^{(h)}: G^{(h)} d x-\int_{\Omega} \varphi \tilde{E}^{(h)}: \tilde{G}^{(h)} d x \rightarrow 0 .
$$

To prove this, we exploit that the most dangerous term in this difference, namely $E^{(h)}:\left(\tilde{G}^{(h)}-G^{(h)}\right)$ has an (approximate) div-curl structure. Finally, we need to pass to the limit in (2.23). The difficulty is that at this point we only know strong convergence of $\tilde{E}^{(h)}$ and not of $E^{(h)}$. To control the remainder term, we first use the fact that $\left(\theta^{(h)}\right)^{\prime}$ cannot concentrate a finite amount of $L^{2}$-norm on the set where the truncation deviates from the original function. To estimate the skew-symmetric part of $E^{h}$ we use its representation (2.19) in connection with another application of the div-curl lemma.

\section{Step 1. Definition of the truncated functions.}


We consider the functions $z^{(h)}$ defined in (2.8) and their rescalings $\check{z}^{(h)}(x):=$ $z^{(h)}\left(x_{1}, x_{2} / h\right)$. Applying Lemma 3.1 to $\check{z}^{(h)}$ with $a=h^{-5 / 8}, A=h^{-7 / 8}$ and undoing the rescaling, we obtain functions $\tilde{z}^{(h)}: \Omega \rightarrow \mathbb{R}^{2}$ and $\lambda_{h} \in\left[h^{-5 / 8}, h^{-7 / 8}\right]$ with the following properties:

$$
\begin{gathered}
\left\|\nabla_{h} \tilde{z}^{(h)}\right\|_{L^{\infty}} \leq \lambda_{h}, \\
\lambda_{h}^{2} \mathcal{L}^{2}\left(A_{h}\right) \leq \frac{C}{\ln (1 / h)} \int_{\Omega}\left|\nabla_{h} z^{(h)}\right|^{2} d x \\
\leq \frac{C}{\ln (1 / h)} \int_{\Omega}\left(\left|G^{(h)}\right|^{2}+\left|\left(\theta^{(h)}\right)^{\prime}\right|^{2}\right) d x,
\end{gathered}
$$

where $A_{h}:=\left\{x \in \Omega: z^{(h)}(x) \neq \tilde{z}^{(h)}(x)\right\}$. In particular, we have

$$
h^{1 / 2} \lambda_{h} \rightarrow \infty, \quad h \lambda_{h} \rightarrow 0, \quad \text { and } \quad \lambda_{h}^{2} \mathcal{L}^{2}\left(A_{h}\right) \rightarrow 0 .
$$

We can also define a sequence of approximated deformations $\tilde{y}^{(h)}: \Omega \rightarrow \mathbb{R}^{2}$ which are associated with the auxiliary functions $\tilde{z}^{(h)}$ :

$$
\tilde{y}^{(h)}:=h \tilde{z}^{(h)}+\int_{0}^{x_{1}} R^{(h)}(s) e_{1} d s+h x_{2} R^{(h)} e_{2} .
$$

Let $\tilde{G}^{(h)}: \Omega \rightarrow \mathbb{M}^{2 \times 2}$ be the corresponding approximated strains defined by the relation

$$
\nabla_{h} \tilde{y}^{(h)}=R^{(h)}\left(I d+h \tilde{G}^{(h)}\right),
$$

and let $\tilde{E}^{(h)}: \Omega \rightarrow \mathbb{M}^{2 \times 2}$ be the corresponding stresses defined as

$$
\tilde{E}^{(h)}:=\frac{1}{h} D W\left(I d+h \tilde{G}^{(h)}\right) .
$$

Using the definition of $\tilde{y}^{(h)}$, it is easy to see that

$$
\tilde{G}^{(h)}=\left(R^{(h)}\right)^{T} \nabla_{h} \tilde{z}^{(h)}-x_{2}\left(\theta^{(h)}\right)^{\prime} e_{1} \otimes e_{1} .
$$

From (3.5) and (3.6), we easily see that $\nabla_{h} \tilde{z}^{(h)}$ and $\nabla_{h} z^{(h)}$ are bounded in $L^{2}$. In fact, they have the same weak limit. To see this, fix $\eta \in L^{2}\left(\Omega ; \mathbb{M}^{2 \times 2}\right)$. Then

$$
\left|\int_{\Omega} \eta:\left(\nabla_{h} \tilde{z}^{(h)}-\nabla_{h} z^{(h)}\right) d x\right| \leq C\left(\int_{A_{h}}|\eta|^{2} d x\right)^{1 / 2} \rightarrow 0 .
$$

Thus

$$
\tilde{G}^{(h)} \rightarrow G \quad \text { weakly in } L^{2}\left(\Omega ; \mathbb{M}^{2 \times 2}\right) .
$$

Step 2. $L^{\infty}$-convergence of $h \tilde{G}^{(h)}$ and strong convergence of sym $\tilde{G}^{(h)}$ and $\tilde{E}^{(h)}$.

We recall the estimate

$$
\sup |f-\bar{f}|^{2} \leq 2\|f\|_{L^{2}}\left\|f^{\prime}\right\|_{L^{2}}, \quad \text { where } \bar{f}=\frac{1}{L} \int_{0}^{L} f d x,
$$

which follows from the identity $\left(g^{2}\right)^{\prime}=2 g g^{\prime}$, applied with $g=f-\bar{f}$. Since $h\left(\theta^{(h)}\right)^{\prime \prime}$ and $\left(\theta^{(h)}\right)^{\prime}$ are bounded in $L^{2}(0, L)$ by (2.3), we have in particular that $(1 / L) \int_{0}^{L}\left|\left(\theta^{(h)}\right)^{\prime}\right| \leq C$ and thus (3.11) yields $\left|\left(\theta^{(h)}\right)^{\prime}\right| \leq C h^{-1 / 2}$. This estimate and inequality (3.5) imply

$$
h\left|\tilde{G}^{(h)}\right| \leq C h \lambda_{h}+C h^{1 / 2} \rightarrow 0 .
$$


Here, we used that $h \lambda_{h} \rightarrow 0$. Expanding $D W$ around the identity as in (2.31), we obtain

$$
\tilde{E}^{(h)}: \tilde{G}^{(h)}=\mathcal{L} \tilde{G}^{(h)}: \tilde{G}^{(h)}+\frac{1}{h} \eta\left(h \tilde{G}^{(h)}\right): \tilde{G}^{(h)},
$$

where the last term on the right-hand side can be bounded by

$$
\frac{1}{h}\left|\eta\left(h \tilde{G}^{(h)}\right): \tilde{G}^{(h)}\right| \leq \omega\left(h\left\|\tilde{G}^{(h)}\right\|_{L^{\infty}}\right)\left|\tilde{G}^{(h)}\right|^{2}
$$

with $\omega(t) \rightarrow 0$, as $t \rightarrow 0^{+}$. Together with (3.12), we obtain for every $\varphi \in C^{\infty}([0, L])$

$$
\int_{\Omega} \varphi \mathcal{L} \tilde{G}^{(h)}: \tilde{G}^{(h)} d x-\int_{\Omega} \varphi \tilde{E}^{(h)}: \tilde{G}^{(h)} d x \rightarrow 0 .
$$

We now show that

$$
\int_{\Omega} \varphi \tilde{E}^{(h)}: \tilde{G}^{(h)} d x-\int_{\Omega} \varphi E^{(h)}: G^{(h)} d x \rightarrow 0 .
$$

Together with the convergence of energy (2.29), the weak convergence of $\tilde{G}^{(h)}$ to $G$ and (3.13) this implies that

$$
\lim _{h \rightarrow 0} \int_{\Omega} \varphi \mathcal{L}\left(\tilde{G}^{(h)}-G\right):\left(\tilde{G}^{(h)}-G\right) d x=0
$$

for every $\varphi \in C^{\infty}([0, L])$ with $\varphi(0)=0$, and hence

$$
\operatorname{sym}\left(\tilde{G}^{(h)}-G\right) \rightarrow 0 \quad \text { strongly in } L^{2}\left((a, L) \times\left(-\frac{1}{2}, \frac{1}{2}\right) ; \mathbb{M}^{2 \times 2}\right)
$$

for all $a>0$. Using again a Taylor expansion we easily deduce that

$$
\tilde{E}^{(h)} \rightarrow E \quad \text { strongly in } L^{2}\left((a, L) \times\left(-\frac{1}{2}, \frac{1}{2}\right) ; \mathbb{M}^{2 \times 2}\right) .
$$

To prove (3.14), we write the difference as

$$
\int_{\Omega} \varphi E^{(h)}:\left(\tilde{G}^{(h)}-G^{(h)}\right) d x+\int_{\Omega} \varphi\left(\tilde{E}^{(h)}-E^{(h)}\right): \tilde{G}^{(h)} d x .
$$

The first term can be controlled by the div-curl lemma; indeed, equalities (3.9) and (2.10) yield

$$
R^{(h)}\left(\tilde{G}^{(h)}-G^{(h)}\right)=\nabla_{h}\left(\tilde{z}^{(h)}-z^{(h)}\right),
$$

so that, by the Euler-Lagrange equation (2.16), we have

$$
\begin{aligned}
& \int_{\Omega} \varphi E^{(h)}:\left(\tilde{G}^{(h)}-G^{(h)}\right) d x=\int_{\Omega} \varphi R^{(h)} E^{(h)}: \nabla_{h}\left(\tilde{z}^{(h)}-z^{(h)}\right) d x \\
& =h \int_{\Omega} \varphi g \cdot\left(\tilde{z}^{(h)}-z^{(h)}\right) d x-\int_{\Omega} \varphi^{\prime} R^{(h)} E^{(h)} e_{1} \cdot\left(\tilde{z}^{(h)}-z^{(h)}\right) d x .
\end{aligned}
$$

Since the sequence $\tilde{z}^{(h)}-z^{(h)}$ converges to 0 strongly in $L^{2}\left(\Omega ; \mathbb{R}^{2}\right)$ and $R^{(h)} E^{(h)}$ is bounded in $L^{2}\left(\Omega ; \mathbb{M}^{2 \times 2}\right)$, we conclude that

$$
\lim _{h \rightarrow 0} \int_{\Omega} \varphi E^{(h)}:\left(\tilde{G}^{(h)}-G^{(h)}\right) d x=0 .
$$

To estimate the second term in (3.17), we use that $\tilde{E}^{(h)}$ and $E^{(h)}$ are bounded in $L^{2}\left(\Omega ; \mathbb{M}^{2 \times 2}\right)$. Thus using Hölder's inequality, we find

$$
\int_{\Omega}\left|\varphi\left(\tilde{E}^{(h)}-E^{(h)}\right): \tilde{G}^{(h)}\right| d x \leq\left(\int_{A_{h}}\left|\tilde{G}^{(h)}\right|^{2} d x\right)^{1 / 2} \leq C\left[\left(\lambda_{h}^{2}+h^{-1}\right) \mathcal{L}^{2}\left(A_{h}\right)\right]^{1 / 2}
$$


and the right-hand side converges to zero in view of (3.7). This concludes the proof of (3.14).

Step 3. Passage to the limit in the Euler-Lagrange equation.

To pass to the limit in (2.23), i.e., in the equation

$$
\int_{0}^{L}\left(\hat{E}_{11}^{(h)} \phi^{\prime}+\left(\theta^{(h)}\right)^{\prime} \hat{E}_{21}^{(h)} \phi+\frac{1}{h} \bar{E}_{21}^{(h)} \phi+\frac{1}{h}\left(\bar{E}_{12}^{(h)}-\bar{E}_{21}^{(h)}\right) \phi\right) d x_{1}=0
$$

for all $\phi \in C^{\infty}([0, L])$ which vanishes on an interval $(0, a)$, we first prove that

$$
\lim _{h \rightarrow 0} \int_{0}^{L}\left(\theta^{(h)}\right)^{\prime} \hat{E}_{21}^{(h)} \phi d x_{1}=0 .
$$

Indeed, it follows from the definition of $\hat{E}^{(h)}$ that

$$
\begin{aligned}
& \int_{0}^{L}\left(\theta^{(h)}\right)^{\prime} \hat{E}_{21}^{(h)} \phi d x_{1}=\int_{\Omega} x_{2}\left(\theta^{(h)}\right)^{\prime} E_{21}^{(h)} \phi d x \\
& \quad=\int_{\Omega} x_{2}\left(\theta^{(h)}\right)^{\prime} \tilde{E}_{21}^{(h)} \phi d x+\int_{A_{h}} x_{2}\left(\theta^{(h)}\right)^{\prime}\left(E_{21}^{(h)}-\tilde{E}_{21}^{(h)}\right) \phi d x .
\end{aligned}
$$

Using (3.16) and the fact that $E_{21}=0$ a.e. in $\Omega$, we obtain that the first integral of the right-hand side converges to 0. As for the second term, using Hölder's inequality, we obtain

$$
\int_{A_{h}}\left|x_{2}\left(\theta^{(h)}\right)^{\prime}\left(E_{21}^{(h)}-\tilde{E}_{21}^{(h)}\right) \phi\right| d x \leq C\left(\int_{A_{h}}\left|\left(\theta^{(h)}\right)^{\prime}\right|^{2} d x\right)^{1 / 2} .
$$

Since $\left|\left(\theta^{(h)}\right)^{\prime}\right| \leq C h^{-1 / 2}$, and $h^{-1} \mathcal{L}^{2}\left(A_{h}\right) \rightarrow 0$ by (3.7), this implies that the second integral on the right-hand side of (3.20) also converges to 0 . This proves (3.19).

Using again a div-curl argument, we will show that

$$
\lim _{h \rightarrow 0} \int_{0}^{L} \frac{1}{h}\left(\bar{E}_{12}^{(h)}-\bar{E}_{21}^{(h)}\right) \phi d x_{1}=0
$$

for every $\phi \in C^{\infty}([0, L])$ which vanishes on $(0, a)$.

Indeed, by (2.19) we have

$$
\frac{1}{h} \text { skew } E^{(h)}=- \text { skew }\left(E^{(h)}\left(G^{(h)}\right)^{T}\right) .
$$

Hence by (2.10)

$$
\frac{1}{h} \text { skew } E^{(h)}=- \text { skew }\left(E^{(h)}\left(\nabla_{h} z^{(h)}\right)^{T} R^{(h)}\right)+x_{2}\left(\theta^{(h)}\right)^{\prime} \text { skew }\left(E^{(h)} e_{1} \otimes e_{1}\right) .
$$

We now use the fact that for $R \in S O(2)$ and $A \in \mathbb{M}^{2 \times 2}$ we have skew $A=$ $R($ skew $A) R^{T}=$ skew $\left(R A R^{T}\right)$ to deduce that

$\frac{1}{h}\left(E_{12}^{(h)}-E_{21}^{(h)}\right)=-\left(R^{(h)} E^{(h)}\right)_{1 j}\left(\nabla_{h} z^{(h)}\right)_{2 j}+\left(R^{(h)} E^{(h)}\right)_{2 j}\left(\nabla_{h} z^{(h)}\right)_{1 j}-x_{2}\left(\theta^{(h)}\right)^{\prime} E_{21}^{(h)}$.

Thus

$$
\begin{aligned}
& \int_{0}^{L} \frac{1}{h}\left(\bar{E}_{12}^{(h)}-\bar{E}_{21}^{(h)}\right) \phi d x_{1} \\
= & \int_{\Omega}\left(-\left(R^{(h)} E^{(h)}\right)_{1 j}\left(\nabla_{h} z^{(h)}\right)_{2 j}+\left(R^{(h)} E^{(h)}\right)_{2 j}\left(\nabla_{h} z^{(h)}\right)_{1 j}\right) \phi d x \\
& -\int_{0}^{L}\left(\theta^{(h)}\right)^{\prime} \hat{E}_{21}^{(h)} \phi d x_{1} .
\end{aligned}
$$


The last term converges to zero by (3.19). The Euler-Lagrange equation (2.16) and the strong convergence of $z^{(h)}$ imply that the remaining terms converge to

$$
\begin{gathered}
\int_{\Omega}(R E)_{11} z_{2} \phi^{\prime}-(R E)_{21} z_{1} \phi^{\prime} d x \\
=\int_{0}^{L}(R \bar{E})_{11} z_{2} \phi^{\prime}-(R \bar{E})_{21} z_{1} \phi^{\prime} d x_{1}=0,
\end{gathered}
$$

where we have used the fact that $z$ is independent of $x_{2}$ and $\bar{E}=0$. This proves (3.21) and together with (3.19) this shows that we can pass to the limit in (3.18). Thus, we obtain again (2.37) and the proof can be concluded as before.

\section{Auxiliary Results}

In this section, we collect and prove some auxiliary results which were needed in the proof of Theorem 1.1 .

We begin with an approximation result for deformations having elastic energy of order $h^{2}$ by means of smooth rotations. This is the point where the rigidity theorem by Friesecke, James, and Müller 4 plays a crucial role (note that in two dimensions the proof of this result can be streamlined using complex variables).

Proposition 4.1. Let $\left(u^{(h)}\right) \subset W^{1,2}\left(\Omega ; \mathbb{R}^{2}\right)$ be a sequence such that

$$
F^{(h)}\left(u^{(h)}\right):=\int_{\Omega} \operatorname{dist}^{2}\left(\nabla_{h} u^{(h)}, S O(2)\right) d x \leq C h^{2},
$$

for every $h>0$. Then there exists an associated sequence $\left(R^{(h)}\right) \subset C^{\infty}\left((0, L) ; \mathbb{M}^{2 \times 2}\right)$ such that

$$
\begin{gathered}
R^{(h)}\left(x_{1}\right) \in S O(2) \quad \text { for every } x_{1} \in(0, L), \\
\left\|\nabla_{h} u^{(h)}-R^{(h)}\right\|_{L^{2}} \leq C h, \\
\left\|\left(R^{(h)}\right)^{\prime}\right\|_{L^{2}}+h\left\|\left(R^{(h)}\right)^{\prime \prime}\right\|_{L^{2}} \leq C
\end{gathered}
$$

for every $h>0$. If, in addition, $u^{(h)}\left(0, x_{2}\right)=\left(0, h x_{2}\right)$, then

$$
\left|R^{(h)}(0)-I d\right| \leq C \sqrt{h} .
$$

Proof. The argument follows closely the proof of Theorem 2.1 in [10]. We include the details for the convenience of the reader.

For every $h>0$ let $k_{h}$ be an integer such that $h \leq \frac{L}{k_{h}}<2 h$. For every $a \in$ $[0, L) \cap \frac{L}{k_{h}} \mathbb{N}$, we define

$$
I_{a, h}:=\left(a, a+\frac{L}{k_{h}}\right) .
$$

We apply the rigidity estimate [4, Theorem 3.1] to the functions $v^{(h)}\left(z_{1}, z_{2}\right):=$ $u^{(h)}\left(z_{1}, z_{2} / h\right)$ restricted to the set $(a, a+2 h) \times\left(-\frac{h}{2}, \frac{h}{2}\right)$ when $a<L-\frac{L}{k_{h}}$ and restricted to the set $(L-2 h, L) \times\left(-\frac{h}{2}, \frac{h}{2}\right)$, otherwise. Thus, we obtain piecewise constant maps $Q^{(h)}:[0, L] \rightarrow S O(2)$ such that

$$
\int_{I_{a, h} \times\left(-\frac{1}{2}, \frac{1}{2}\right)}\left|\nabla_{h} u^{(h)}-Q^{(h)}\right|^{2} d x \leq C \int_{(a, a+2 h) \times\left(-\frac{1}{2}, \frac{1}{2}\right)} \operatorname{dist}^{2}\left(\nabla_{h} u^{(h)}, S O(2)\right) d x .
$$


When $a=L-\frac{L}{k_{h}}$, replace the interval $(a, a+2 h)$ by $(L-2 h, L)$ in the second integral above. We point out that the constant $C$ above is independent of $h$. Summing over $a$, we obtain

$$
\int_{\Omega}\left|\nabla_{h} u^{(h)}-Q^{(h)}\right|^{2} d x \leq C F^{(h)}\left(u^{(h)}\right) \leq C h^{2} .
$$

Let $a \in[0, L) \cap \frac{L}{k_{h}} \mathbb{N}$ be such that $(a, a+4 h) \subset(0, L)$ and let $b:=a+\frac{L}{k_{h}}$. Then, using estimate (4.5), its analog for the set $(a, a+4 h) \times\left(-\frac{1}{2}, \frac{1}{2}\right)$, and the fact that both intervals $I_{a, h}, I_{b, h}$ are contained in $(a, a+4 h)$, we have

$$
\frac{L}{k_{h}}\left|Q^{(h)}(a)-Q^{(h)}(b)\right|^{2} \leq C \int_{(a, a+4 h) \times\left(-\frac{1}{2}, \frac{1}{2}\right)} \operatorname{dist}^{2}\left(\nabla_{h} u^{(h)}, S O(2)\right) d x .
$$

In particular, we deduce that

$$
\left|Q^{(h)}\left(x_{1}+s\right)-Q^{(h)}\left(x_{1}\right)\right|^{2} \leq C h^{-1} F^{(h)}\left(u^{(h)}\right) \leq C h
$$

for every $x_{1} \in(h, L-h)$ and every $|s| \leq h$. If we extend $Q^{(h)}$ by $Q^{(h)}(0)$ for $x_{1}<0$ and by $Q^{(h)}(L)$ for $x_{1}>L$, estimate (4.8) holds for all $x_{1} \in \mathbb{R}$.

Iterative application of inequality (4.7) provides a difference quotient estimate for $Q^{(h)}$. More precisely,

$$
\int_{\mathbb{R}}\left|Q^{(h)}\left(x_{1}+s\right)-Q^{(h)}\left(x_{1}\right)\right|^{2} d x_{1} \leq C h^{-2}(|s|+h)^{2} F^{(h)}\left(u^{(h)}\right) \leq C(|s|+h)^{2} .
$$

Let $\eta \in C_{0}^{\infty}(0,1)$ be such that $\eta \geq 0$ and $\int_{0}^{1} \eta(s) d s=1$. We set $\eta_{h}(s):=\frac{1}{h} \eta\left(\frac{s}{h}\right)$ and define

$$
\tilde{Q}^{(h)}\left(x_{1}\right):=\int_{0}^{h} \eta_{h}(s) Q^{(h)}\left(x_{1}-s\right) d s, \quad x_{1} \in[0, L] .
$$

Using estimate (4.9), we easily see that

$$
\begin{gathered}
\left\|\tilde{Q}^{(h)}-Q^{(h)}\right\|_{L^{2}} \leq C h, \\
\left\|\left(\tilde{Q}^{(h)}\right)^{\prime}\right\|_{L^{2}} \leq C, \quad h\left\|\left(\tilde{Q}^{(h)}\right)^{\prime \prime}\right\|_{L^{2}} \leq C
\end{gathered}
$$

for every $h>0$.

Let $\pi: U \rightarrow S O(2)$ be a smooth projection from a neighbourhood $U$ of $S O(2)$ onto $S O(2)$. Since from (4.8) and Jensen's inequality we have that

$$
\left\|\tilde{Q}^{(h)}-Q^{(h)}\right\|_{L^{\infty}}^{2} \leq C h,
$$

the functions $\tilde{Q}^{(h)}$ take values in $U$ for $h$ small enough. Therefore, we can define $R^{(h)}:=\pi\left(\tilde{Q}^{(h)}\right)$. Properties (4.1)-(4.3) follow immediately from (4.6) and (4.10).

To establish (4.4), we start from the following trace inequality

$$
\int_{\left(-\frac{1}{2}, \frac{1}{2}\right)}\left|v\left(0, z_{2}\right)-\bar{v}\right|^{2} d z_{2} \leq C \int_{(0, l) \times\left(-\frac{1}{2}, \frac{1}{2}\right)}|\nabla v|^{2} d z,
$$

which holds uniformly for $1 \leq l \leq 2$, with $\bar{v}=\int v\left(0, z_{2}\right) d z_{2}$. We apply this estimate with $v(z)=h^{-1} u^{(h)}\left(h z_{1}, z_{2}\right)-\bar{Q} z$, where $\bar{Q}=Q^{(h)}(0)$. In combination with (4.5) at $a=0$ and the boundary condition for $u^{(h)}$, this yields

$$
\int_{\left(-\frac{1}{2}, \frac{1}{2}\right)}\left(\left|\bar{Q}_{12} x_{2}\right|^{2}+\left|x_{2}-\bar{Q}_{22} x_{2}\right|^{2}\right) d x_{2} \leq C h .
$$

Since $\bar{Q} \in S O(2)$, this implies that $|\bar{Q}-I d| \leq C \sqrt{h}$. In view of (4.11), this yields (4.4). 
Proposition 4.2. Assume that the energy density $W$ is differentiable and its derivative $D W$ is Lipschitz continuous. Assume moreover that $D W$ is differentiable at the identity. Suppose that

$$
G^{(h)} \rightarrow G \quad \text { weakly in } L^{2}\left(\Omega ; \mathbb{M}^{2 \times 2}\right)
$$

and define the rescaled stresses as in 2.14) by

$$
E^{(h)}:=\frac{1}{h} D W\left(I d+h G^{(h)}\right) .
$$

Then

$$
E^{(h)} \rightarrow E:=\mathcal{L} G \quad \text { weakly in } L^{2}\left(\Omega ; \mathbb{M}^{2 \times 2}\right),
$$

where $\mathcal{L}:=D^{2} W(I d)$.

Proof. Since $\left(E^{(h)}\right)$ is bounded in $L^{2}\left(\Omega ; \mathbb{M}^{2 \times 2}\right)$, it is enough to show that the limit of each weakly convergent subsequence of $\left(E^{(h)}\right)$ coincides with $\mathcal{L} G$. Therefore, let $E^{\left(h_{k}\right)}$ converge weakly in $L^{2}\left(\Omega ; \mathbb{M}^{2 \times 2}\right)$ to some $\tilde{E}$.

A Taylor expansion of $D W$ around the identity yields

$$
\frac{1}{h} D W(I+h A)=\mathcal{L} A+\frac{1}{h} \eta(h A),
$$

where $|\eta(F)| /|F| \rightarrow 0$ as $|F| \rightarrow 0$. Set $\omega(t):=\sup \{|\eta(F)| /|F|:|F| \leq t\}$ for every $t>0$. Then $\omega(t) \rightarrow 0$, as $t \rightarrow 0^{+}$. Applying formula (4.15) with $A$ replaced by $G^{\left(h_{k}\right)}$ and $h$ replaced by $h_{k}$, we find that

$$
E^{\left(h_{k}\right)}=\frac{1}{h_{k}} D W\left(I d+h_{k} G^{\left(h_{k}\right)}\right)=\mathcal{L} G^{\left(h_{k}\right)}+\frac{1}{h_{k}} \eta\left(h_{k} G^{\left(h_{k}\right)}\right)
$$

for every $k$. Now let $M_{k}:=\left\{x \in \Omega:\left|G^{\left(h_{k}\right)}(x)\right| \leq h_{k}^{-1 / 2}\right\}$, and let $\chi_{k}$ be its characteristic function. Since $\chi_{k} \rightarrow 1$ boundedly in measure and $G^{(h)} \rightarrow G$ weakly in $L^{2}\left(\Omega ; \mathbb{M}^{2 \times 2}\right)$, we have

$$
\chi_{k} E^{\left(h_{k}\right)} \rightarrow \tilde{E} \quad \text { and } \quad \chi_{k} G^{\left(h_{k}\right)} \rightarrow G \quad \text { weakly in } L^{2}\left(\Omega ; \mathbb{M}^{2 \times 2}\right) .
$$

Moreover, from (4.16) it follows that for every $k$

$$
\chi_{k} E^{\left(h_{k}\right)}=\mathcal{L}\left(\chi_{k} G^{\left(h_{k}\right)}\right)+\frac{1}{h_{k}} \chi_{k} \eta\left(h_{k} G^{\left(h_{k}\right)}\right) .
$$

The first term on the right-hand side converges weakly in $L^{2}\left(\Omega ; \mathbb{M}^{2 \times 2}\right)$ to $\mathcal{L} G$ by (4.17). As for the second term, we have

$$
\frac{1}{h_{k}} \chi_{k}\left|\eta\left(h_{k} G^{\left(h_{k}\right)}\right)\right| \leq \omega\left(\sqrt{h_{k}}\right)\left|G^{\left(h_{k}\right)}\right|
$$

where we used the fact that $h_{k}\left|G^{\left(h_{k}\right)}\right| \leq \sqrt{h_{k}}$ on $M_{k}$. Since $\omega\left(\sqrt{h_{k}}\right)$ converges to 0 and $\left|G^{\left(h_{k}\right)}\right|$ is bounded in $L^{2}\left(\Omega ; \mathbb{M}^{2 \times 2}\right)$, we conclude that the second term on the right-hand side of (4.18) converges to 0 strongly in $L^{2}\left(\Omega ; \mathbb{M}^{2 \times 2}\right)$. Passing to the limit in (4.18) and using (4.17), we finally obtain $\tilde{E}=\mathcal{L} G$, so that the proposition is proved.

We conclude this section by proving the truncation lemma stated and used in Section 3 . We first consider a fixed domain in $\mathbb{R}^{n}$ and then extend the result to the thin domains $\Omega_{h}$ by successive reflection. 
Lemma 4.3. Let $1<p<\infty, n \geq 2, m \geq 1, n, m \in \mathbb{N}$ and let $\Omega$ be a bounded Lipschitz domain in $\mathbb{R}^{n}$ or $\Omega=\mathbb{R}^{n}$. Then there exists a constant $C_{2}$ (depending on $\Omega, p, n$, and $m$ ) such that for every $A>a>0$ and every function $v: \Omega \rightarrow \mathbb{R}^{m}$ with $\nabla v \in L^{p}\left(\Omega ; \mathbb{M}^{m \times n}\right)$ there exist $\lambda \in[a, A]$ and a function $v^{\lambda}: \Omega \rightarrow \mathbb{R}^{m}$ such that

$$
\begin{aligned}
\left|\nabla v^{\lambda}\right| & \leq \lambda, \\
\lambda^{p}\left|\left\{v^{\lambda} \neq v\right\}\right| & \leq \frac{C_{2}}{\ln (A / a)} \int_{\Omega}|\nabla v|^{p} d x .
\end{aligned}
$$

Remark 4.4. Estimate (4.20) is useful, if $A$ is much larger than $a$. If $A$ and $a$ are comparable one can use the simpler estimate $\lambda^{p}\left|\left\{v^{\lambda} \neq v\right\}\right| \leq C_{2}|| \nabla v \|_{p}^{p}$ for all $\lambda>0$, which even holds for $p=1$, see, e.g., [3].

Proof. This is a simple consequence of the standard results on the truncation of gradients, see, e.g., [7, 15, 3. We sketch the argument for the convenience of the reader. For simplicity, we only consider the scalar case $m=1$, the general case can easily be treated by using the maximal function of $|\nabla v|$ and extending each component of $v$ separately. We first observe that it suffices to prove that there exists constants $C_{1}^{\prime}$ and $C_{2}^{\prime}$ such that one can always find $\lambda \in[a, A]$ with the following properties

$$
\begin{aligned}
\left|\nabla v^{\lambda}\right| & \leq C_{1}^{\prime} \lambda \\
\lambda^{p}\left|\left\{v^{\lambda} \neq v\right\}\right| & \leq \frac{C_{2}^{\prime}}{\ln (A / a)} \int_{\Omega}|\nabla v|^{p} d x .
\end{aligned}
$$

Indeed, if (4.21) and (4.22) hold for $w$ and $w^{\lambda}$ (in place of $v$ and $v^{\lambda}$ ) take $w=C_{1}^{\prime} v$ and $v^{\lambda}:=w^{\lambda} / C_{1}^{\prime}$. Then we obtain (4.19) and (4.20) with $C_{2}:=C_{2}^{\prime}\left(C_{1}^{\prime}\right)^{p}$.

We first consider the case $\Omega=\mathbb{R}^{n}$. Let $f$ denote the Hardy-Littlewood maximal function of $\nabla v$,

and let

$$
f(x):=\sup _{R>0} \frac{1}{|B(x, R)|} \int_{B(x, R)}|\nabla v| d y
$$

$$
E^{t}:=\left\{x \in \mathbb{R}^{n}: f(x)>t\right\} .
$$

By standard estimates for the maximal function, we have

$$
\|f\|_{L^{p}} \leq C_{3}\|\nabla v\|_{L^{p}}
$$

where $C_{3}$ depends only on $p$. From the definition of $E^{t}$ and Poincaré inequality, one can easily deduce that (see, e.g., 3], p. 253)

$$
|v(x)-v(y)| \leq C_{4} t|x-y|, \quad \text { for a.e. } x, y \notin E^{t} .
$$

Hence (after removal of a null set), $v$ is Lipschitz in the complement of $E^{t}$ and hence has an extension $v^{t}$ with the same Lipschitz constant. Thus

$$
\begin{aligned}
\left|\nabla v^{t}\right| & \leq C_{4} t, \\
\left|\left\{v \neq v^{t}\right\}\right| & \leq\left|E^{t}\right| .
\end{aligned}
$$

From the definition of $E^{t}$, we have the trivial estimate $t^{p}\left|E^{t}\right| \leq\|f\|_{p}^{p} \leq C_{3}^{p}|| \nabla v \|_{p}^{p}$. To obtain the refined estimate (4.20), we use the relation

$$
\int_{0}^{\infty} p t^{p-1}\left|E^{t}\right| d t=\int_{\mathbb{R}^{n}} f^{p} d x
$$


Set $g(t):=t^{p}\left|E^{t}\right|$. Then

$$
\int_{a}^{A} \frac{p}{t} \inf _{t \in[a, A]} g(t) d t \leq \int_{a}^{A} \frac{p}{t} g(t) d t \leq \int_{\mathbb{R}^{n}}|f|^{p} d x \leq C_{3}^{p} \int_{\mathbb{R}^{n}}|\nabla v|^{p} d x .
$$

This yields (4.20) with $C_{2}\left(\mathbb{R}^{n}\right)=\frac{1}{p} C_{3}^{p}$.

Suppose that $\Omega$ is a bounded Lipschitz domain. We may assume that $\int_{\Omega} v=0$ (otherwise we first define $w:=v-m$, where $m$ is the average of $v$, apply the result for $w$ and finally set $v^{\lambda}:=w^{\lambda}+m$ ). Then Poincaré's inequality yields

$$
\|v\|_{W^{1, p}(\Omega)} \leq C_{5}\|\nabla v\|_{L^{p}(\Omega)} \text {. }
$$

Thus there exists an extension $\tilde{v}: \mathbb{R}^{n} \rightarrow \mathbb{R}^{m}$ with (see, e.g., [13])

$$
\|\tilde{v}\|_{W^{1, p}\left(\mathbb{R}^{n}\right)} \leq C_{6}\|v\|_{W^{1, p}(\Omega)} \leq C_{7}\|\nabla v\|_{L^{p}(\Omega)} .
$$

Now we can apply the previous reasoning to $\tilde{v}$ and we get (4.19) and (4.20) with $C_{2}(\Omega)=C_{2}\left(\mathbb{R}^{n}\right) C_{7}^{p}$.

Proof of Lemma 3.1. Let $h>0$ and let $u \in W^{1,2}\left(\Omega_{h} ; \mathbb{R}^{2}\right)$. First of all, note that $u$ can be extended to the rectangle $\Omega=(0, L) \times\left(-\frac{1}{2}, \frac{1}{2}\right)$ by successive reflection. By Lemma 4.3 there exist $\lambda \in[a, A]$ and $w \in W^{1, \infty}\left(\Omega ; \mathbb{R}^{2}\right)$ such that

$$
\|\nabla w\|_{L^{\infty}(\Omega)} \leq \lambda
$$

and

$$
\lambda^{2} \mathcal{L}^{2}(\{x \in \Omega: u(x) \neq w(x)\}) \leq \frac{C}{\ln (A / a)} \int_{\Omega}|\nabla u|^{2} d x .
$$

Let $N_{h}$ be the largest integer such that $h N_{h}+h / 2 \leq 1 / 2$. For $i \in \mathbb{Z} \cap\left[-N_{h}, N_{h}\right]$ let $S_{h, i}:=(0, L) \times\left(i h-\frac{h}{2}, i h+\frac{h}{2}\right)$ and let

$$
R_{h}:=\Omega \backslash \bigcup_{-N_{h} \leq i \leq N_{h}} S_{h, i}
$$

Since

$$
\sum_{-N_{h} \leq i \leq N_{h}} \mathcal{L}^{2}\left(\{u \neq w\} \cap S_{h, i}\right) \leq \mathcal{L}^{2}(\{u \neq w\}),
$$

there exists some index $i_{0}$ such that

$$
\begin{aligned}
\lambda^{2} \mathcal{L}^{2}\left(\{u \neq w\} \cap S_{h, i_{0}}\right) & \leq \frac{1}{2 N_{h}+1} \lambda^{2} \mathcal{L}^{2}(\{u \neq w\}) \\
& \leq \frac{C}{2 N_{h}+1} \frac{1}{\ln (A / a)} \int_{\Omega}|\nabla u|^{2} d x .
\end{aligned}
$$

Let $v: \Omega_{h} \rightarrow \mathbb{R}^{2}$ be the function defined by

$$
v\left(x_{1}, x_{2}\right):=w\left(x_{1}, i_{0} h+(-1)^{i_{0}} x_{2}\right) \quad \text { for every }\left(x_{1}, x_{2}\right) \in \Omega_{h} .
$$

It is clear that $v \in W^{1, \infty}\left(\Omega_{h} ; \mathbb{R}^{2}\right)$ and that it satisfies (3.1) by (4.23). Moreover, since $u$ has been extended to $\Omega$ by reflection, we have

$$
\left\{x \in \Omega_{h}: u(x) \neq v(x)\right\}=\left\{x \in S_{h, i_{0}}: u(x) \neq w(x)\right\}
$$

and

$$
\int_{\Omega}|\nabla u|^{2} d x \leq\left(2 N_{h}+3\right) \int_{\Omega_{h}}|\nabla u|^{2} d x .
$$

Now assertion (3.2) follows from (4.25) -4.27). This finishes the proof of Lemma 3.1 


\section{ACKNOWLEDGEMENTS}

We would like to thank R. Pakzad for many helpful comments. M.G.M. and S.M. were supported by the Marie Curie research training network MRTN-CT2004-505226 (MULTIMAT). M.G.M. was also partially supported by MIUR project

"Calculus of Variations" 2004.

\section{REFERENCES}

[1] S. Conti, Habilitation thesis. University of Leipzig, 2003.

[2] S. Conti and F. Maggi, Confining thin elastic sheets and folding paper. Preprint, http://analysis.math.uni-duisburg.de/publications/, 2005.

[3] L.C. Evans and R.F. Gariepy, Measure theory and fine properties of functions. CRC Press, 1992.

[4] G. Friesecke, R.D. James and S. Müller, A theorem on geometric rigidity and the derivation of nonlinear plate theory from three dimensional elasticity. Comm. Pure Appl. Math. 55 (2002), 1461-1506.

[5] G. Friesecke, R.D. James and S. Müller, A hierarchy of plate models, derived from nonlinear elasticity by Gamma-convergence. Arch. Rational Mech. Anal. 180 (2006), 183-236.

[6] H. LeDret and A. Raoult, The nonlinear membrane model as a variational limit of nonlinear three-dimensional elasticity. J. Math. Pures Appl. 73 (1995), 549-578.

[7] F.C. Liu, A Lusin type property of Sobolev function. Indiana Univ. Math. J. 26 (1977), 645651.

[8] A. Lobkovsky, S. Gentges, H. Li, D. Morse and T.A. Witten, Scaling properties of stretching ridges in a crumpled elastic sheet. Science 270 (1995), 1482-1485.

[9] A. Mielke, On Saint Venant's problem for an elastic strip. Proc. Roy. Soc. Edinburgh Sect. A 110 (1988), 161-181.

[10] M.G. Mora and S. Müller, Derivation of the nonlinear bending-torsion theory for inextensible rods by $\Gamma$-convergence. Calc. Var. Partial Differential Equations 18 (2003), 287-305.

[11] O. Pantz, Une justification partielle du modèle de plaque en flexion par $\Gamma$-convergence. C.R. Acad. Sci. Paris Sér. I Math. 332 (2001), 587-592.

[12] O. Pantz, On the justification of the nonlinear inextensional plate model. Arch. Rational Mech. Anal. 167 (2003), 179-209.

[13] E. Stein, Singular integrals and differentiability properties of functions. Princeton UP, 1970.

[14] S.C. Venkataramani, Lower bounds for the energy in a crumpled elastic sheet - a minimal ridge. Nonlinearity 17 (2004), 301-312.

[15] W. Ziemer, Weakly differentiable functions. Springer, 1989.

(M.G. Mora) SiSSA, Via Beirut 2-4, 34014 Trieste, Italy

(S. Müller) Max Planck Institute for Mathematics in the Sciences, Inselstrasse 22, 04103 LeipZig, Germany

(M.G. Schultz) Max Planck Institute for Mathematics in the Sciences, Inselstrasse 22, 04103 LEIPZIG, Germany

E-mail address, Maria Giovanna Mora: mora@sissa.it

E-mail address, Stefan Müller: sm@mis.mpg.de

E-mail address, Maximilian Schultz: schultz@mis.mpg.de 